\title{
Adolescent cannabis use, baseline prodromal symptoms and the risk of psychosis
}

Antti Mustonen, Solja Niemelä, Tanja Nordström, Graham K. Murray, Pirjo Mäki, Erika Jääskeläinen and Jouko Miettunen

\section{Background}

The association between cannabis use and the risk of psychosis has been studied extensively but the temporal order still remains controversial.

\section{Aims}

To examine the association between cannabis use in adolescence and the risk of psychosis after adjustment for prodromal symptoms and other potential confounders.

\section{Method}

The sample ( $n=6534)$ was composed of the prospective general population-based Northern Finland Birth Cohort of 1986. Information on prodromal symptoms of psychosis and cannabis use was collected using questionnaires at age 15-16 years. Participants were followed up for ICD-10 psychotic disorders until age 30 years using nationwide registers.

\section{Results}

The risk of psychosis was elevated in individuals who had tried cannabis five times or more (hazard ratio, $(\mathrm{HR})=6.5,95 \% \mathrm{Cl} 3.0$ 13.9). The association remained statistically significant even when adjusted for prodromal symptoms, other substance use and parental psychosis $(\mathrm{HR}=3.0,95 \% \mathrm{Cl} 1.1-8.0)$.

\section{Conclusions}

Adolescent cannabis use is associated with increased risk of psychosis even after adjustment for baseline prodromal symptoms, parental psychosis and other substance use.

\section{Declaration of interest}

None.

\section{Copyright and usage}

(c) The Royal College of Psychiatrists 2018.
Increasing evidence points towards a dose-response relationship between the level of cannabis use and the risk for psychotic outcomes, ${ }^{1-4}$ and also indicates that cannabis use results in earlier onset of psychosis. ${ }^{5,6}$ Most longitudinal studies have taken reverse causation into account by excluding individuals with pre-existing psychotic symptoms, which could have led to overestimates of the true association. ${ }^{7}$ Moreover, most of these previous studies have focused on psychotic symptoms rather than psychotic illness per se. Several studies have examined the impact of psychotic baseline severity in relation to the cannabis use-psychosis association (for example Arseneault et al, Henquet et al, Kelley et al and Bechtold et al). ${ }^{8-11}$ To our knowledge, there is only one prospective study examining cannabis use and psychosis diagnosis (schizophreniform syndrome) that has taken baseline psychotic (prodromal) symptoms into account as a potential confounder before or at the time of cannabis use. ${ }^{8}$ Furthermore, cannabis use often co-occurs with other types of substance use, which may also have an impact on the psychosis outcome. ${ }^{12}$

The aim of this study was to examine the predictive association between adolescent cannabis use at age 15-16 years and the risk of subsequent psychosis by the age of 30 years in the Northern Finland Birth Cohort Study 1986 (NFBC1986). An association between cannabis use and prodromal symptoms in adolescence has been reported previously in NFBC1986. ${ }^{13}$ We hypothesised that cannabis use increases the risk for psychosis independently of baseline prodromal symptoms, parental psychosis and other forms of substance use including daily tobacco smoking.

\section{Method}

\section{Participants}

NFBC1986 is an ongoing follow-up study including $99 \%$ of all births, comprising all the live born children $(n=9432)$ from the two northernmost provinces in Finland. ${ }^{14}$ In all, $7344(48.8 \%$ boys) participated in the follow-up study in 2001-2002, when the participants were aged 15-16 years. Participants who signed the informed consent form and answered the questions on cannabis use were included in the present study. Adolescents who had received a psychosis diagnosis before the 15-16-year follow-up $(n=10)$ were excluded from the study. The final sample included 6534 individuals $(49.1 \%$ boys). Information on psychosis-related diagnoses was collected from the national registries until the end of 2015, i.e. by the age of 30 years. The study was approved by the ethics committee of the Northern Ostrobothnia Hospital District in Finland (17 May 2006). The data flow is shown in Fig. 1.

\section{Data collection}

Data collection started prospectively before birth and has continued since. ${ }^{14}$ A multidisciplinary NFBC field study was conducted when the adolescents were 15-16 years of age. Data on substance misuse was collected in 2001-2002, in two different surveys. First, they received a postal questionnaire, which included questions on smoking habits. Thereafter, all the participants were invited to a field study where they completed self-report questionnaires including questions on prodromal symptoms for psychosis (PRODscreen), ${ }^{15}$ on alcohol use and illicit substance use. For more detailed information about the study and its design, see Fig. 1 (see also the NFBC1986 webpage, available at http://www.oulu.fi/nfbc/node/ 40696).

\section{Measures}

\section{Cannabis use}

The data on adolescent cannabis use was collected using questionnaires that the participants received during the field study when they were aged 15-16 years. The participants were asked 'Have you ever used marihuana or hashish?' as dichotomised (no/yes) and with options: (a) never, (b) once, (c) 2-4 times, (d) 5 times or 


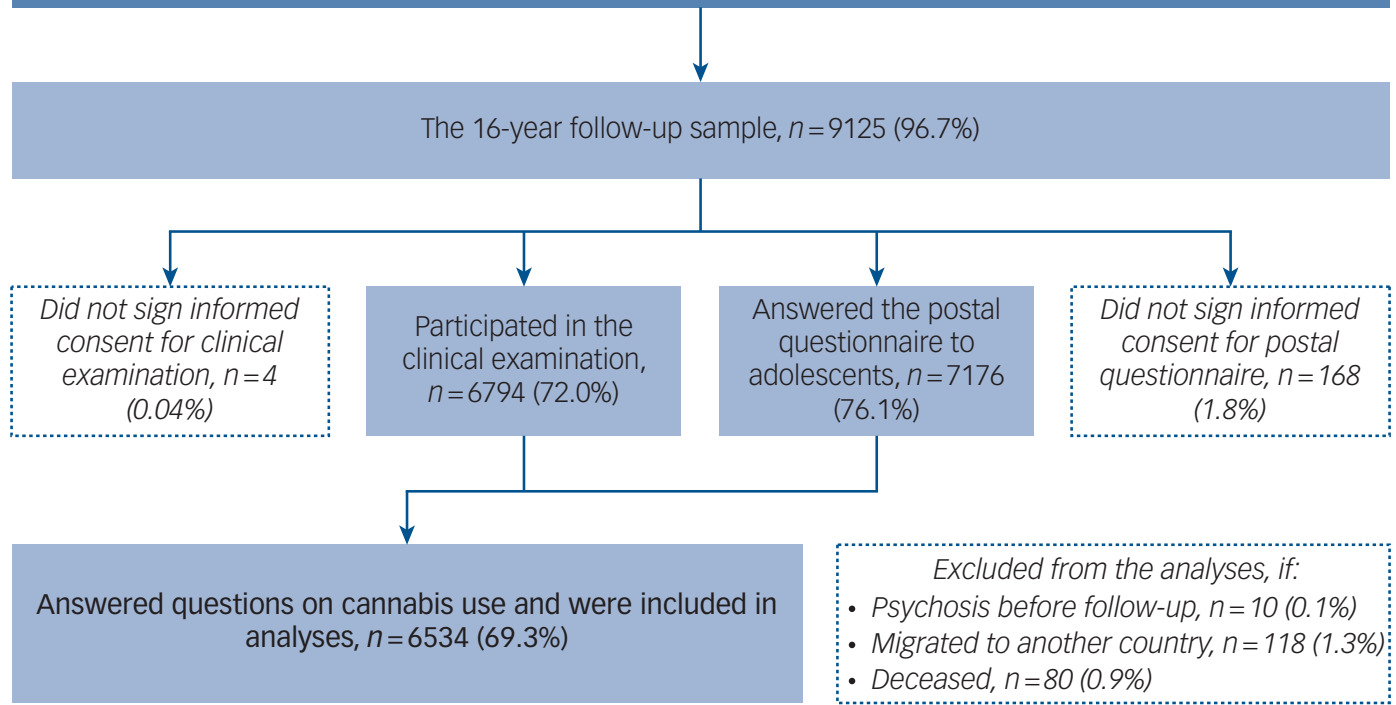

Fig. 1 Data flow from the current study using the Northern Finland Birth Cohort 1986

more, or (e) regularly. Options (d) and (e) were pooled because of small sample size in the two categories. ${ }^{13}$

\section{Psychosis diagnoses}

Information on diagnosed non-organic psychoses (ICD-10: F20, F22-F29, F302, F312, F315, F323, F333) was gathered from nationwide registers: the Care Register for Health Care 2001-2015 and the Register of Primary Health Care Visits 2011-2015 of the National Institute for Health and Welfare, disability pensions of the Finnish Centre for Pensions 2001-2015, and the medication reimbursement register of the Social Insurance Institution of Finland 2001-2005. The Care Register contains data on patients discharged from in-patient care, and since 1998 also data on specialised outpatient care. The Register of Primary Health Care Visits covers all out-patient primary healthcare delivered in Finland. For more comprehensive information about the registers see Supplement 1 in Filatova et al. ${ }^{16}$ Age at the time when psychosis was first detected was estimated as the onset age of psychosis. Cumulative incidence of psychoses was calculated.

\section{Prodromal symptoms}

When the cohort members were 15-16 years old the participants were asked about the occurrence of prodromal symptoms during the previous 6 months (no/yes) using the PROD-screen ${ }^{15}$. The PROD-questionnaire has 12 specific items (no/yes) rating, for example feelings that something strange or inexplicable is taking place within oneself or in the environment, feelings that one is being followed or influenced in some special way, experience of thoughts running wild or difficulty in controlling the speed of thoughts. The sum of the questionnaire was used as a continuous variable in the analyses.

\section{Smoking and substance use other than cannabis}

Data on tobacco smoking, alcohol use and use of illicit drugs or other intoxicants was collected at two points during the 15-16year follow-up: information on regular tobacco smoking was ascertained in postal questionnaires and other data on substance use were collected in the questionnaire that the participants received during the field study. Daily tobacco smoking was studied with the question 'Are you currently smoking tobacco daily (no/yes)'. Frequent drunkenness was questioned as a subjective measure 'Have you been drunk during the past year $(0,1-2,3-5,6-9,10-19,20-39$ or 40 times or more)', but we categorised this as 'Have you been drunk during the past year 10 times or more (no/yes)'. Data were also collected on any use of other substances with several questions (no/yes), for example prescription drug use, use of inhalants and illicit drugs. Because of the low number of users in each group, these were combined as 'Other substance use (no/yes)'.

\section{Family structure}

Information on family structure was gathered by combining information collected from parents at birth and when the cohort member was aged 15-16. The classification of the family pattern included families with (a) both parents living with the participant all the time (intact families) and (b) other (non-intact) families. ${ }^{13}$

\section{Place of residence}

Data were collected based on the population density of the residential area at age 15-16 years in order to account for possible confounding due to place of residence. The variable was dichotomised in the analyses (urban $v$. non-urban).

\section{Socioeconomic status of the family}

The socioeconomic status of the family was estimated by the highest education level achieved by either parent when the child was aged 15-16. This variable was categorised as: professionals (professionals, entrepreneurs and other white-collar workers) and non-professionals. ${ }^{13}$

\section{Parental psychosis}

Information on parental psychosis (no/yes) was based on parental diagnoses in the nationwide registers. These registers include: (a) Register of Health Care during the years 1972-2015 (Hospital Discharge Register until 1994). This register also includes visits to 
specialised out-patient healthcare since 1998; (b) disability pensions of the Finnish Centre for Pensions (1965-2013); and (c) The Register of Primary Health Care Visits (2011-2015).

\section{Statistical methods}

We used logistic regression analysis with Cox-regression analysis (hazard ratios (HRs) with 95\% confidence intervals) to assess the effect of cannabis use on the risk of psychosis. Times of emigration and death were used as censoring points in the analyses. We also conducted additional Cox-regression analyses on cannabis use and separate psychosis diagnoses. Cannabis use was included in the analyses as dichotomised (no/yes) and categorised (never, once, 2-4 times, 5 times or more). Logistic regression analysis with odds ratios (ORs with 95\% confidence intervals) were used to study the associations between (a) covariates (i.e. family characteristics, smoking, alcohol use and other drug use) and cannabis use (dichotomised), and (b) covariates and psychosis diagnosis. We also studied dose-response with a trend test, in which cannabis use was entered into the regression analysis as a continuous variable. In the final Cox-regression analyses, we included covariates that had statistically significant effects $(P<0.05)$ on both the outcome of cannabis use and psychosis diagnosis. Additionally, we also included parental psychosis (no/yes) because it is known to be a potential risk factor for psychosis although it reached statistical significance only for psychosis risk and thus did not fulfil the inclusion criteria as described above. All statistical analyses were performed using SPSS 21.0.

\section{Results}

\section{Background variables, cannabis use and occurrence of prodromal symptoms and psychosis}

The background variables under study and their relation to cannabis use and occurrence of psychosis during the follow-up are presented in Table 1. In all, there were 375 (5.7\%) ever users of cannabis of which $66(1.0 \%)$ had used cannabis more than five times. Girls reported cannabis use more commonly than boys (6.3\% v. 5.1\%, $P<0.05)$. Among those who had tried cannabis once, $58.9 \%$ were female $(112 / 190)$. The respective figures for $2-4$ times and 5 times or more were $55.5 \%$ (66/119) and 48.5\% (32/ 66). Daily smokers were more likely to use cannabis than nondaily smokers $(22.2 \%$ v. $3.3 \%, P<0.05$, see Table 1$)$.

In total, 1993 adolescents (30.5\% of the study sample) reported a frequency of three or more items on PROD-screen. Altogether 124 individuals with psychoses emerged in the sample population (29 narrow-defined schizophrenia, 10 schizophrenia spectrum disorder (schizoaffective disorder and delusional disorder), 12 bipolar disorder with psychotic episodes, 19 major depression with psychotic features, 54 other psychosis (brief reactive psychosis, other psychotic disorder). Of these, 57 out of 124 (46.0\%) were female.

\section{Attrition}

In the attrition analysis, participants from non-intact families were less likely to participate in the study than individuals from intact families $(60 \% v$. $70 \%)$ when the participants were $15-16$ years old.

Table 1 Family characteristics, smoking, alcohol use and other drug use by cannabis-use categories in the 15- to 16-year follow-up study of the Northern Finland 1986 Birth Cohort

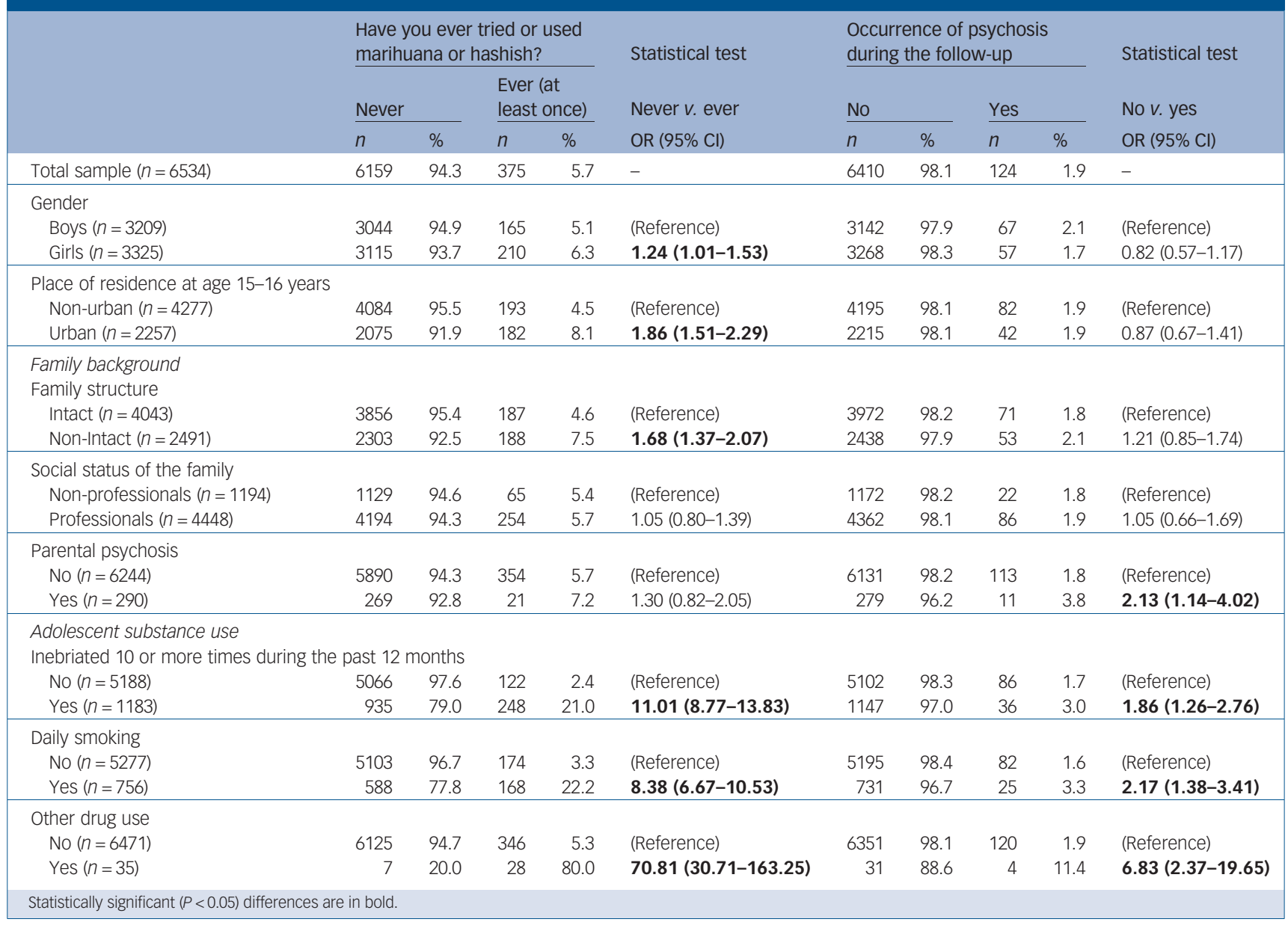


Table 2 The hazard ratios (HR) for the risk of psychosis in Northern

Finland Birth Cohort 1986 in different groups of cannabis use ${ }^{a}$

\begin{tabular}{|c|c|c|}
\hline Cannabis use & $n$ & $\mathrm{HR}(95 \% \mathrm{Cl})$ \\
\hline \multicolumn{3}{|l|}{ Crude $(n=6534)$} \\
\hline Never & 6159 & (Reference) \\
\hline Ever & 375 & $2.85(1.73-4.67)$ \\
\hline \multicolumn{3}{|l|}{ Crude $(n=6534)$} \\
\hline Never & 6159 & (Reference) \\
\hline Once & 190 & $1.53(0.63-3.76)$ \\
\hline 2-4 times & 119 & $3.03(1.33-6.90)$ \\
\hline 5 times or more & 66 & $6.47(3.01-13.91)$ \\
\hline \multicolumn{3}{|l|}{ Model $1(n=6534)$} \\
\hline Never & 6159 & (Reference) \\
\hline Once & 190 & $1.21(0.49-2.98)$ \\
\hline 2-4 times & 119 & $2.25(0.98-5.18)$ \\
\hline 5 times or more & 66 & $4.38(2.00-9.59)$ \\
\hline \multicolumn{3}{|l|}{ Model $2(n=5872)$} \\
\hline Never & 5534 & (Reference) \\
\hline Once & 171 & $1.13(0.44-2.90)$ \\
\hline 2-4 times & 108 & $1.43(0.50-4.07)$ \\
\hline 5 times or more & 59 & $3.16(1.21-8.29)$ \\
\hline \multicolumn{3}{|l|}{ Model $3(n=5872)$} \\
\hline Never & 5534 & (Reference) \\
\hline Once & 171 & $1.15(0.46-2.95)$ \\
\hline 2-4 times & 108 & $1.46(0.51-4.16)$ \\
\hline 5 times or more & 59 & $3.02(1.14-7.98)$ \\
\hline \multicolumn{3}{|c|}{$\begin{array}{l}\text { Statistically significant }(P<0.05) \text { differences are in bold. } \\
\text { a. Covariates: Model 1: } P R O D \text {; Model 2: PROD-screen other substance use HR }=2.19 \\
(0.67-7.17) \text {, frequent alcohol use } H R=1.27(0.78-2.07) \text {, daily tobacco } \text { smoking } H R=1.42 \\
(0.84-2.39) \text {; Model 3: Model 2, parental psychosis } H R=1.83(0.91-3.64) \text {. }\end{array}$} \\
\hline
\end{tabular}

Also, participation rate in individuals from non-urban area $(72 \%)$ was higher than in those from urban areas $(64 \%) .{ }^{13}$ Not participating in the follow-up study was associated with greater risk for psychosis $(\mathrm{HR}=1.52,95 \%$ CI 1.13-2.04).

\section{Associations between adolescent cannabis use and subsequent} psychosis

The frequencies and hazard ratios for the risk of psychosis in relation to cannabis use are presented in Table 2. Of the cannabis users, 18 out of $375(4.8 \%)$ received a psychosis diagnosis during the 15-year follow-up (4 narrow-defined schizophrenia, 4 schizophrenia spectrum disorder, 0 bipolar disorder with psychotic features, 7 major depression with psychotic features, 3 other psychosis). Among those without adolescent cannabis use, the incidence of psychosis was $1.7 \%$ ( $n=106 ; 25$ narrow-defined schizophrenia, 6 schizophrenia spectrum disorder, 12 major depression with psychotic features, 12 bipolar disorder with psychotic features, 51 other psychosis).

Use of cannabis at the age 15-16 years was associated with psychosis (unadjusted $\mathrm{HR}=2.85 ; 95 \%$ CI 1.73-4.67). When studied by category, using cannabis less than five times had no statistically significant association with psychosis. The proportion of psychoses in each cannabis-use category were as follows: once $2.6 \%(5 / 190), 2-4$ times $5.0 \%(6 / 119)$ and for five times or more $10.6 \%(7 / 66)$. A dose-response was seen with the trend test $(\mathrm{OR}=1.83$; 95\% CI 1.45-2.31). More frequent use of cannabis, i.e. using at least five times, was associated with subsequent psychosis $(\mathrm{HR}=6.47 ; 3.01-13.91)$, and this was also evident when the data was adjusted for prodromal symptoms of psychosis (Table 2). When further adjusted for daily tobacco smoking, frequent alcohol use, other substance use and parental psychosis, the association between frequent cannabis use in this sample and subsequent psychosis attenuated but remained statistically significant (Table 2).

When the psychosis diagnoses were analysed separately, diagnoses of psychotic depression (HR $=9.74,95 \%$ CI 3.83-24.73) and

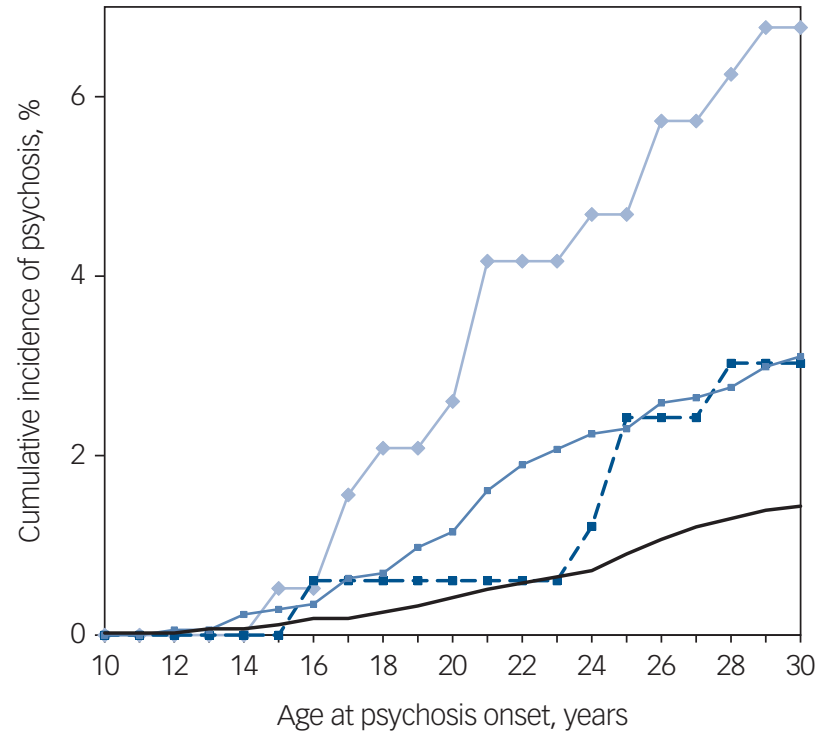

$\ldots$ Cannabis use + and prodromal symptoms of psychosis $+(n=13 / 134)$

- Cannabis use + and prodromal symptoms of psychosis $-(n=5 / 134)$

$\rightarrow$ Cannabis use - and prodromal symptoms of psychosis $+(n=54 / 134)$

- Cannabis use - and prodromal symptoms of psychosis $-(n=62 / 134)$

Fig. 2 Cumulative incidences of psychosis in four groups with and without cannabis use and prodromal symptoms in the Northern Finland Birth Cohort 1986.

schizophrenia spectrum disorder $(\mathrm{HR}=11.18,95 \%$ CI 3.16-39.62) were associated with cannabis use. No statistical significance was reached for schizophrenia, bipolar disorder with psychotic episodes and other psychosis in cannabis users $v$. controls.

Figure 2 presents cumulative incidences of psychosis and hazard curves for psychosis risk by cannabis use and prodromal symptoms in four groups. As can be seen in this descriptive figure, adolescents with prodromal symptoms (cut off $\geq 3$ ) and cannabis use had approximately a twofold higher incidence of psychosis by age 30 years than adolescents with prodromal symptoms but no cannabis use.

\section{Discussion}

\section{Main findings}

Cannabis use at the age of 15-16 years was associated with subsequent psychosis diagnosis, and this was evident in the group with heaviest cannabis use even after controlling for baseline prodromal symptoms, daily smoking, frequent alcohol use, other substance use and parental psychosis. We found a dose-response effect suggesting that more frequent cannabis use is associated with a greater risk for psychosis.

Our results further confirm the previous results of metaanalyses $^{1-4}$ indicating that adolescent cannabis use is associated with increased risk for psychosis. In our study, this was evident even when adjusted with multiple covariates. Further, in line with previous studies, ${ }^{1,3}$ cannabis use was associated with subsequent psychosis diagnosis with a dose-response effect.

\section{Comparison with findings from other studies}

As stated earlier, several studies have examined the impact of psychotic baseline severity in relation to the cannabis-psychosis association (for example, Arseneault et al, Henquet et al, Kelley 
et al and Bechtold et al). ${ }^{8-11}$ but to our knowledge there is only one prospective study (Dunedin) examining cannabis use and a psychosis diagnosis that has adjusted for psychotic baseline severity. ${ }^{8}$ In our study, the association between the use of cannabis five times or more in adolescence and subsequent psychosis remained statistically significant after adding the prodromal symptoms to the multivariable model. However, inclusion of the prodromal symptoms decreased the hazard ratio of the cannabis-psychosis association approximately by $30 \%$. There might be a subsample of participants that are self-medicating, but it is unlikely that the whole decrease in the strength of association would be because of self-medicating since the study population was only 15- to 16-years olds during the field study. Some studies have revealed certain schizophrenia risk alleles are associated with increased use of cannabis. ${ }^{17}$ In addition, a recent study revealed that the association between symptoms of cannabis-use disorder and psychotic-like experiences in young adults could be partly explained by shared genetic factors. ${ }^{18}$ Therefore, the decrease in the strength of the association might rather be because of the high correlation between these two variables (PROD-screen $>3$ and cannabis use). However, shared genetic factors likely explain only part of the association and we are still missing the data from adolescent samples.

In our previous report those who had tried cannabis had a higher mean number of prodromal symptoms than the controls (3.11 v. $1.88 ; t$-test $8.68, P<0.001) .{ }^{13}$ Experiencing prodromal symptoms in adolescence is common ${ }^{19}$ and in our study $30.5 \%$ of adolescents reported a frequency of three or more items in the PROD-screen. It should be noted that as this is a populationbased study, individuals have self-reported prodromal symptoms and therefore differ from clinical or other high-risk samples ${ }^{20}$ since no interview assessing symptoms was performed. It is likely that the current estimate of frequency of prodromal symptoms would have been much lower in an interview. For example, in the Avon Longitudinal Study of Parents and Children, 38.9\% of children self-reported experiencing prodromal symptoms in the previous 6 months; however, observer-rated assessments identified only $13.7 \%$ of children as experiencing these symptoms. ${ }^{21}$

In our current study, participants with prodromal symptoms and cannabis use in adolescence had double the incidence of psychosis compared with controls with prodromal symptoms but no cannabis use (Fig. 2). Current evidence indicates that early-onset cannabis use predates the onset of psychosis, especially among those with pre-existing vulnerability and heavier cannabis use. $^{22}$ Subjects with prodromal symptoms in adolescence might be more prone to adverse effects of cannabis, experiencing more severe prodromal and psychotic symptoms compared with those without cannabis use.

In line with previous strong evidence, ${ }^{23}$ parental psychosis was a significant risk factor for offspring psychosis. However, frequent cannabis use in adolescence increased the risk for psychotic illness independently of parental psychosis in our sample. Participants with both parental psychosis and cannabis use have been shown to have a greater risk for psychotic outcomes. ${ }^{23}$ It has been speculated that there might be a shared genetic aetiology between cannabis dependence and schizophrenia, ${ }^{24}$ which may in part explain the associations between adolescent cannabis use, parental psychosis and onset of offspring psychotic illness. Unfortunately, because of the relatively low number of individuals with psychosis among the cannabis users, we could not reliably study the interaction of these risk factors (parental psychosis and adolescent cannabis use).

Cannabis-use disorder has been associated with greater psychosis conversion, independently of other forms of substance use. $^{12}$ In order to study the cannabis-specific association with psychotic illness, we added daily smoking, frequent drunkenness or illicit drug use other than cannabis to the multivariable models with parental psychosis history. In these analyses, an association between frequent cannabis use and psychosis was shown independent of other substance use. However, statistical significance between frequent cannabis use and subsequent psychotic illness was attenuated by $28 \%$ when substance use other than cannabis, i.e. daily smoking, frequent drunkenness or other forms of substance use, was included in the multivariate model with prodromal symptoms. This may mostly be explained by overadjustment, because polysubstance use was very common among cannabis users. For example, $45 \%$ of the cannabis users were also daily smokers and $66 \%$ of cannabis users were frequent alcohol users. Furthermore, assessing the true significance of cannabis use or cigarette smoking may also be challenging because cannabis users usually smoke cannabis mixed with tobacco. ${ }^{25}$

We reported that individuals from non-intact families $(60 \% v$. $70 \%)$ and urban areas (64\% v. 72\%) were less likely to participate in the NFBC field study in 2001-2002. As these individuals were more likely to have ever used cannabis, it is likely that our proportion of individuals using cannabis is underestimated, but as the participation rates are still very good it is unlikely that this affected the overall conclusions of the study. Although boys report cannabis use more often in general, ${ }^{26}$ here girls reported more cannabis use than boys $(6.3 \%$ v. $5.1 \%)$. However, this was mostly because of more sporadic use than in boys. It can be speculated that girls of this age (15-16 years) might be more prone to single experiments with cannabis because of earlier puberty. Finally, although there were more cannabis users among girls, gender did not reach statistical significance for psychosis in the crude analyses. Unfortunately, we were unable to analyse psychosis risks separately by gender because of the low number of cannabis users.

We reported also that specifically diagnoses of psychotic depression and schizophrenia spectrum disorder were associated with cannabis use. Interestingly, no significance was found for narrow schizophrenia. As a result of the small number of cases strong conclusions cannot be drawn, since we could not analyse the effect of frequent use of cannabis on separate diagnoses. However, this has not been reported in previous prospective samples to our knowledge and should be noted in further studies.

\section{Strengths and limitations}

There are notable strengths in our study.

(a) This is one of the largest birth cohort studies with high genetic and ethnic homogeneity with relatively low attrition.

(b) We were able to use several nationwide registers, and only a very small proportion $(2.1 \%)$ of cohort members died or emigrated during the follow-up. Therefore, the coverage of clinically significant psychosis diagnoses within this population during the 15-year-follow-up can be considered to be high.

(c) We made substantial efforts to minimise the possibility of residual confounding.

(d) Furthermore, adjusting for prodromal symptoms indicated a possible temporal or even causal link between cannabis use and subsequent psychosis that is in line with previous research.

However, the following limitations should be taken into account when interpreting our results.

(a) The number of cannabis users with a psychosis diagnosis was relatively low. Also, no prospective data on cannabis use before or after the age of 15-16 years was available. 
(b) Information on substance use was collected using self-report, which may give rise to a bias of underreporting and possible underestimation of the true association. ${ }^{7}$

(c) Cannabinoid levels of cannabis may vary considerably, and different strains may have different psychotropic potentials. ${ }^{27}$ The THC/CBD concentration of different strains has also increased considerably over the years ${ }^{28}$ and may alter the generalizability of our results. This should be noted in future studies.

(d) Unfortunately, we did not have any data on other family/relatives with psychosis and this should be noted in order to account for genetic vulnerability more comprehensively.

(e) Individuals from non-intact families and urban areas were less likely to participate in the NFBC field study in 2001-2002, which may introduce bias. Further, adolescents with prodromal symptoms may also be less likely to answer on selfreport questionnaires, as the risk of psychosis was higher among the non-participants compared with those who participated.

\section{Implications}

Our study provides further evidence on the temporal order of adolescent cannabis use and psychosis. According to our results, cannabis use increases the risk for subsequent psychosis even after prodromal symptoms of psychosis, parental psychosis and other substance use have been taken into account. However, it is still controversial whether cannabis use alone is a sufficient risk factor for psychosis or rather a component cause in the pathogenesis. The importance of psychoeducation and prevention of adolescent cannabis use is emphasised as adolescents using cannabis and having prodromal symptoms were at twofold risk for subsequent psychosis.

Antti Mustonen, MD, Center for Life Course Health Research, University of Oulu, Oulu, Finland and Medical Research Center Oulu, Oulu University Hospital and University of Oulu, Oulu, Finland; Solja Niemelä, MD, PhD, Department of Psychiatry, Research Unit of Clinical Neuroscience, University of Oulu, Oulu, Finland and Department of Psychiatry, Lapland Hospital District, Rovaniemi, Finland; Tanja Nordström, PhD, Center for Life course Health Research, University of Oulu, Oulu, Finland and Medical Research Center Oulu, Oulu University Hospital and University of Oulu, Oulu, Finland; Graham K. Murray, MRCPSych, Department of Psychiatry, University of Cambridge, Cambridge, UK;

Pirjo Mäki, MD, PhD, Medical Research Center Oulu, Oulu University Hospital and University of Oulu, Oulu, Finland and Department of Psychiatry, Research Unit of Clinical Neuroscience, University of Oulu, Oulu, Finland and Department of Psychiatry, Oulu University Hospital, the Northern Ostrobothnia Hospital District, Oulu, Finland and Department of Psychiatry, Länsi-Pohja healthcare district, Finland and Department of Psychiatry, the Middle Ostrobothnia Central Hospital, Soite, Finland and Mental health services, Joint Municipal Authority of Wellbeing in Raahe District, Finland and Mental PhD, Jouko Miettunen, PhD, Center for Life Course Health Research, University of Oulu, Oulu, Finland and Medical Research Center Oulu, Oulu University Hospital and University of Oulu, Oulu, Finland.

Correspondence: Antti Mustonen, Center for Life Course Health Research, University of Oulu, P.O. Box 5000, 90014 University of Oulu, Finland. Email: antti.mustonen@student. oulu.fi

First received 1 Dec 2016, final revision 26 Oct 2017, accepted 23 Nov 2017

\section{Funding}

This study was funded by EU QLG1-CT-2000-01643 (EUROBLCS) Grant no. E51560, NorFA Grant no. 731, 20056, 30167, USA/NIHH 2000 G DF682 Grant no. 50945, the Academy of Finland (\#268336), Jalmari and Rauha Ahokas Foundation, the Northern Finland Health Care Support Foundation, NARSAD: the Brain and Behavior Research Fund, the Signe and Ane Gyllenberg Foundation, Finland, the Sigrid Jusélius Foundation, Finland, Thule Institute, University of Oulu, Finland and Finnish Cultural Foundation, The Olvi Foundation, Juho Vainio Foundation and Oulun Lääketieteellinen tutkimussäätiö.

\section{Acknowledgements}

We thank Professor Anna-Liisa Hartikainen (for the launching of NFBC1986), the participants in the study and the NFBC project center.

\section{References}

1 Marconi A, Di Forti M, Lewis CM, Murray RM, Vassos E. Meta-analysis of the association between the level of cannabis use and risk of psychosis. Schizophr Bull 2016; 42: 1262-9.

2 Henquet C, Murray R, Linszen D, van Os J. The environment and schizophrenia: the role of cannabis use. Schizophr Bull 2005; 31: 608-12.

3 Moore TH, Zammit S, Lingford-Hughes A, Barnes TR, Jones PB, Burke M, et al. Cannabis use and risk of psychotic or affective mental health outcomes: a systematic review. Lancet 2007; 28: 319-28.

4 Semple DM, McIntosh AM, Lawrie SM. Cannabis as a risk factor for psychosis: systematic review. J Psychopharmacol 2005; 19: 187-94.

5 Large M, Sharma S, Compton MT, Slade T, Nielssen O. Cannabis use and earlier onset of psychosis: a systematic meta-analysis. Arch Gen Psychiatry 2011; 68: 555-61.

6 Myles N, Newall H, Nielssen O, Large M. The association between cannabis use and earlier age at onset (1) of schizophrenia and other psychoses: metaanalysis of possible confounding factors. Curr Pharm Des 2012; 18: 5055-69.

7 Gage SH, Hickman M, Zammit S. Association between cannabis and psychosis: epidemiologic evidence. Biol Psychiatry 2016; 79: 549-56.

8 Arseneault L, Cannon M, Poulton R, Murray R, Caspi A, Moffitt TE. Cannabis use in adolescence and risk for adult psychosis: Iongitudinal prospective study. BMJ 2002; 325: 1212-3.

9 Henquet C, Krabbendam L, Spauwen J, Kaplan C, Lieb R, Wittchen HU, et al. Prospective cohort study of cannabis use, predisposition for psychosis, and psychotic symptoms in young people. BMJ 2005; 330: 11.

10 Kelley ME, Wan CR, Broussard B, Crisafio A, Cristofaro S, Johnson S, et al. Marijuana use in the immediate 5-year premorbid period is associated with increased risk of onset of schizophrenia and related psychotic disorders. Schizophr Res 2016; 17: 62-7.

11 Bechtold J, Hipwell A, Lewis DA, Loeber R, Pardini D. Concurrent and sustained cumulative effects of adolescent marijuana use on subclinical psychotic symptoms. Am J Psychiatry 2016; 173: 781-9.

12 Auther AM, Cadenhead KS, Carrion RE, Addington J, Bearden CE, Cannon TD, et al. Alcohol confounds relationship between cannabis misuse and psychosis conversion in a high-risk sample. Acta Psychiatr Scand 2015; 132: 60-8.

13 Miettunen J, Tormanen S, Murray GK, Jones PB, Maki P, Ebeling H, et al. Association of cannabis use with prodromal symptoms of psychosis in adolescence. Br J Psychiatry 2008; 192: 470-1.

14 Jarvelin MR, Hartikainen-Sorri AL, Rantakallio P. Labour induction policy in hospitals of different levels of specialisation. Br J Obstet Gynaecol 1993; 100: 310-5.

15 Heinimaa M, Salokangas RK, Ristkari T, Plathin M, Huttunen J, llonen T, et al. PROD-screen-a screen for prodromal symptoms of psychosis. Int J Methods Psychiatr Res 2003; 12: 92-104.

16 Filatova S, Marttila R, Koivumaa-Honkanen $\mathrm{H}$, Nordstrom T, Veijola J, Maki $\mathrm{P}$ et al. A comparison of the cumulative incidence and early risk factors for psychotic disorder in young adults in the Northern Finland Birth Cohorts 1966 and 1986. Epidemiol Psychiatr Sci 2016; 28: 1-11.

17 Power RA, Verweij KJ, Zuhair M, Montgomery GW, Henders AK, Heath AC, et al. Genetic predisposition to schizophrenia associated with increased use of cannabis. Mol Psychiatry 2014; 19: 1201-4.

18 Nesvag R, Reichborn-Kjennerud T, Gillespie NA, Knudsen GP, Bramness JG, Kendler KS, et al. Genetic and environmental contributions to the association between cannabis use and psychotic-like experiences in young adult twins. Schizophr Bull 2017; 43: 644-653.

19 Yung AR, Nelson B, Baker K, Buckby JA, Baksheev G, Cosgrave EM. Psychoticlike experiences in a community sample of adolescents: implications for the continuum model of psychosis and prediction of schizophrenia. Aust N Z J Psychiatry 2009; 43: 118-28.

20 Granö N, Karjalainen M, Itkonen A, Anto J, Edlund V, Heinimaa M, et al. Differential results between self-report and interview-based ratings of risk symptoms of psychosis. Early Interv Psychiatry 2011; 5: 309-14.

21 Horwood J, Salvi G, Thomas K, Duffy L, Gunnell D, Hollis C, et al. IQ and nonclinical psychotic symptoms in 12-year-olds: results from the ALSPAC birth cohort. Br J Psychiatry 2008; 193: 185-91.

22 Bagot KS, Milin R, Kaminer Y. Adolescent initiation of cannabis use and earlyonset psychosis. Subst Abus 2015; 36: 524-533.

23 Radhakrishnan R, Wilkinson ST, D'Souza DC. Gone to pot - a review of the association between cannabis and psychosis. Front Psychiatry 2014; 5: 54.

24 Sherva $\mathrm{R}$, Wang $\mathrm{Q}$, Kranzler $\mathrm{H}$, Zhao $\mathrm{H}$, Koesterer $\mathrm{R}$, Herman $\mathrm{A}$, et al. Genome-wide association study of cannabis dependence severity, novel risk variants, and shared genetic risks. JAMA Psychiatry 2016; 73: 472-80.

25 Gage SH, Hickman M, Heron J, Munafo MR, Lewis G, Macleod J, et al. Associations of cannabis and cigarette use with psychotic experiences at age 
18: findings from the Avon Longitudinal Study of Parents and Children. Psychol Med 2014; 44: 3435-44.

26 Thanki D, Matias J, Griffiths $\mathrm{P}$, Noor A, Olszewski D, Simon R, et al Prevalence of Daily Cannabis Use in the European Union and Norway. European Monitoring Centre for Drugs and Drug Addiction (EMCDDA), 2012.
27 Di Forti M, Sallis H, Allegri F, Trotta A, Ferraro L, Stilo SA, et al. Daily use, especially of high-potency cannabis, drives the earlier onset of psychosis in cannabis users. Schizophr Bull 2014; 40: 1509-17.

28 Murray RM, Quigley H, Quattrrone D, Englund A, Di Forti M. Traditional marijuana, high-potency cannabis and synthetic cannabinoids: increasing risk for psychosis. World Psychiatry 2016; 15: 195-204.

\section{poems}

\section{I'm getting there - A patient-inspired poem}

\section{Katherine Murdoch}

At 11, I was standing in the courtroom. Trying to fix you

Emotional breakdowns and abuse were my toys

Trying to fly out of bedroom windows

While my family didn't want the burden of my cloud, in theirs

I became you.

College didn't get on with me

So the hairdressing was gonna be the new start

Everyone wished for me

But hairspray got in my throat, you see

They wanted my smile to beam and my appearance to gleam

But my cloud didn't see the sun

The only shine my body sees is when it's imprinting on my skin, cold

See the imprint is one, which will keep

It scars deep.

And the tiny white mountains on me,

My skin,

Are stared at by climbing eyes

Who could not possibly comprehend their origin

The grey lifeless cloud, which won't just hide away

In another place.

And I know what you think of these lines, so a cocoon of my house is fine

The front door, firmly shut, cut

But the same room in the same house, the walls are mocking me and

My body is imprinted on the sheets like stone, alone

And the little red raindrops are falling to the floor, staining my existence with a plop

But there is no rainbow without rain,

So being able to leave my bed or throwing up everything I consume with the tablets I ingest is the decision to be alive

Not to thrive.

So I'm reaching my hands out for you to see

To see the white mountains on my skin

My mum is angry, she wants you to see and society to believe

The tablets are endless, five times a day, before not after meals

20/30/80 mg of this, that, and stop

They don't work on me

I've tried counselling opening up to who, what, when

So believe me, help me, help me to be the daughter I'm supposed to be.

You breathe,

And tentatively try to solve the soggy puzzle of my mind

That really is quite kind but I'm a bit messed up, up there

The notion of fixing me up in a shiny box, with my name neatly on the front, has gone

Long gone

But I came here to share

I'm getting there.

(c) Katherine Murdoch, reproduced with permission. 